\title{
Power load characterization for Type-I ELMy H-Modes in JET
}

H.Thomsen ${ }^{1}$, T.Eich ${ }^{1}$, S.Devaux ${ }^{1}$, G.Arnoux ${ }^{2}$, S.Brezinsek ${ }^{3}$, E.delaLuna ${ }^{4,5}$, W.Fundamenski ${ }^{2}$, A.Herrmann ${ }^{1}$, A.Huber ${ }^{3}$, S.Jachmich ${ }^{4,6}$, P.Lomas ${ }^{2}$, I.Nunes ${ }^{4,7}$, G.Saibene ${ }^{8}$, A.Scarabosio ${ }^{1}$, J.Schweinzer ${ }^{1}$ and JET EFDA Contributors ${ }^{*}$

JET-EFDA, Culham Science Centre, Abingdon, OX14 3DB, UK

1) Max-Planck-Institut für Plasmaphysik, EURATOM-Association, D-17491 Greifswald, Germany

2) Culham Science Centre, EURATOM/CCFE Fusion Association, Abingdon, OX14 3DB, UK

3) IPP-Energieforschung, Forschungszentrum Jülich GmbH, Association EURATOM, 52425 Jülich, Germany

4) EFDA-CSU, Culham Science Centre, Abingdon, OX14 3DB, UK

5) Laboratorio Nacional de Fusión, Asociación EURATOM-CIEMAT, Madrid, Spain

6) ERM/KMS, Association EURATOM - 30 Avenue de la Renaissance B-1000 Brussels, Belgium

7) Associação EURATOM-IST, Instituto de Plasmas e Fusão Nuclear - L.A., IST, Lisboa, Portugal

8) Fusion for Energy Joint Undertaking, 08019, Barcelona, Spain

E-mail contact of main author: henning.thomsen @ipp.mpg.de

52.55.Fa, 52.55.Rk, 52.40.Hf

\begin{abstract}
.
ELM resolved divertor target power load studies were conducted for a wide range of discharge conditions in the JET tokamak. The magnetic configuration of these discharges was optimized for the fast divertor IR camera observing the outboard target. It is found that the ELM size estimated from the diamagnetic energy is not uniquely determining the ELM energy load at the divertor target. ELM mid plane integral deposited power widths between 7
\end{abstract}

\footnotetext{
* See the Appendix of F. Romanelli et al., Proceedings of the 23rd IAEA Fusion Energy Conference 2010, Daejeon, Korea
} 
$\mathrm{mm}$ and $18 \mathrm{~mm}$ are observed, the inter-ELM widths lie in a range of $2.5 \mathrm{~mm}$ to $6 \mathrm{~mm}$. This ELM broadening is found to widen with ELM size. The temporal evolution of the ELM shape was characterized by rise and decay times. The ELM rise times are found to be in the range expected for ITER $(250 \mu \mathrm{s})$, but the ELM decay is usually larger than assumed for the ITER design.

\section{Introduction}

One critical factor in the standard H-mode scenario of the ITER operation is the maximum allowable energy load for transient events on the plasma facing components. The impact of edge localized modes (ELMs) onto the divertor target plates should stay below an energy load of $0.5 \mathrm{MJ} / \mathrm{m}^{2}$ per ELM (pulse duration $\sim 500 \mu \mathrm{s}$ ) to avoid a significant reduction of the divertor life time due to material eroision [1]. If the predominant deposited ELM energy load were a factor of 2-3 above this limit, a CFC erosion rate of approximately $1 \mu \mathrm{m} / \mathrm{ELM}$ and large scale melting of $\mathrm{W}$ with possible ejection of droplets would occur and could require a replacement of the divertor targets after only 10000 ELMs.

The expected ELM size in ITER is extrapolated from current day devices based on the multimachine $v^{*}$ scaling [2]. The ELM dynamics in the scrape-off layer (SOL) plays an important role for the spatial and temporal characteristics of the ELMs arriving at the divertor and first wall.

Following the installation of a fast high resolution infrared (IR) system viewing at the JET divertor [4] a large number of experiments have been performed which are optimized for power exhaust studies with focus on ELM and inter-ELM profiles. Type-I ELMy H-Mode deuterium plasmas with currents from 1MA-3.8MA, $\mathrm{q}_{95}$ in the range of 3.3-5.4 with low $(\delta=0.25)$ and high magnetic configuration triangularity $(\delta=0.4)$ were investigated.

\section{Setup, Validation and Methods}

The time resolution of the new divertor IR camera installed in the 2008 campaign (FLIR/Titanium, 3-5 $\mu \mathrm{m}$ wave length, typical time resolution $\Delta \mathrm{t}_{\mathrm{IR}}=86 \mu \mathrm{s}$ for a strip of $4 \mathrm{X} 320$ pixels, typical stare time $40 \mu \mathrm{s}$ ) allows for ELM resolved measurements of power loads on the JET outboard divertor target (tile 5 in Fig. 1(a)) with a $1.7 \mathrm{~mm}$ spatial resolution. 
Optical restrictions in the set-up lead to an unfavourable coverage of the inboard target because the viewing angle is too oblique. This results in a spatial resolution of $5 \mathrm{~mm}$ at the inboard target. Especially during ELM times an unknown fraction of the deposited power spills over to the further inboard divertor tile with unknown surface layer properties. In this paper we therefore constrain ourselves to data from the outboard divertor (covered by 150 pixels in radial direction).

The IR based absolute estimate of the surface temperature of the CFC-tiles is validated by comparing with tile embedded divertor thermocouple (DVTC). The discharge integrated energy balance from IR with respect to DVTC is found to lie within a range of $80-120 \%$ as shown in Fig. 1(b). Pyrometer temperature measurements at the same divertor tile are available for some discharges and the agreement with the surface temperature measured by the IR camera is better than $3 \%$. Comparison to divertor Langmuir probe measurements (LP) for selected discharges are used to assess the influence of the divertor target surface thermal properties on the inferred heat fluxes by IR for short events such as ELMs [3]. A detailed comparison between IR and LP measurements for a single ELM event with striations in the heat flux profile across the divertor target resolved by both diagnostics showed an interdiagnostics time difference in the range of the sampling rate of both diagnostics [4].

The non-linear finite-element code THEODOR code was used to calculate the heat fluxes assuming a $2 \mathrm{~d}$ geometry and temperature dependent material parameters [5, 6].

The data are divided into ELM and inter-ELM phases and the power on the divertor target as well as the wetted area are estimated for each ELM and inter-ELM phase ( $\sim 1 \mathrm{~ms}$ before the next ELM). The rise time $\tau_{\text {rise }}$ from the ELM start (steep increase of heat flux) to ELM peak and the decay time $\tau_{\text {decay }}$ from ELM peak to 1/e decay are estimated for each individual ELM (cf. Fig 2).

The heat flux profiles for ELM and inter-ELM phases are of special interest in terms of the plasma surface interaction and there are several methods used in the Literature in order to estimate the spatial decay width in the SOL-region from IR target data [7]. Here, we use the wetted area, which can be mapped into an integral deposited power width considering the magnetic flux expansion from the outer mid plane to the target. The wetted area (in $\mathrm{m}^{2}$ ) is estimated from the ratio between the target-integrated power $\mathrm{P}(\mathrm{W})$ and the peak heat flux $\mathrm{Q}_{\text {peak }}\left(\right.$ in $\left.\mathrm{W} / \mathrm{m}^{2}\right), \mathrm{A}_{\text {wet }}=\mathrm{P} / \mathrm{Q}_{\text {peak }}\left(\mathrm{m}^{2}\right)$ at the time $\mathrm{t}\left(\mathrm{Q}_{\text {peak }}=\mathrm{max}\right)$.

ELM energy loads,

$\varepsilon=1 /(2 \cdot \mathrm{s} 0) \cdot \int_{\mathrm{s}=\mathrm{speak}-\mathrm{s} 0}{ }^{\text {speak }+\mathrm{s} 0}\left(\int_{\mathrm{t}=\mathrm{tELMstart}}{ }^{\text {ELMdecay }} \mathrm{Q}(\mathrm{s}, \mathrm{t}) \mathrm{dt}\right) \mathrm{ds}$ 
where $\mathrm{s}$ is the spatial coordinate along the divertor target in the radially outward direction, are calculated for a poloidal average of $2 . \mathrm{s} 0=1 \mathrm{~cm}$ centered around the position, $\mathrm{s}_{\text {peak }}$, of the ELM peak heat flux, speak. The integration time is from the ELM start (steep increase of heat flux) until the 1/e decay after the ELM peak. Note, that this definition of $\varepsilon$ characterizes the local energy load in contrast to an integral definition via the ratio between target deposited energy and wetted area. In terms of material erosion limits by power transients, the energy load should be more relevant since the erosion will occur localized as well.

For the estimation of the ELM wetted area a second approach can be utilized, the ratio between the energy $(\mathrm{J})$ and the energy load (in $\mathrm{J} / \mathrm{m}^{2}$ ). Since time integrated signals are considered, the latter method should be less affected by an over-estimation of the peak heat flux due to surface layer effects. In fact we find that the second method based on the energy load yields slightly larger values of the wetted area. We show here the wetted area estimates based on the first method $\left(\mathrm{A}_{\mathrm{wet}}=\mathrm{P} / \mathrm{Q}_{\text {peak }}\right)$, therefore, the wetted area estimates should be smaller and thus more conservative.

The wetted area at the divertor targets can be used to estimate a mid plane integral deposited power width $\lambda[7,4]$. Considering the magnetic geometry and flux expansion, the conversion is given by $\lambda=A_{w e t} / 85 \mathrm{~m}$.

To characterize the ELM size we use the energy difference $\Delta \mathrm{W}_{M H D}$ in the plasma stored energy before the ELM and $4 \mathrm{~ms}$ after the drop. The relative ELM size is given by normalization to $\mathrm{W}_{\mathrm{MHD}}$.

The current data base contains a total of 61 time-windows in 52 discharges, time window intervals ranging from $0.5 \mathrm{~s}$ to $12 \mathrm{~s}$. More than 6000 individual ELMs and preceding interELM values are included. In certain discharge conditions the type-I ELMs are accompanied by compound ELM phases [18]. ELMs in these phases are (partly) also detected by the automated software. An assessment of ELMs in the data base by manual selection yields a number of 2647 ELMs in compound phases and 3382 type-I ELMs in the data base.

\section{Temporal characterization of ELM shapes}

The time period in which an ELM deposits its power on the divertor target is an important parameter for the estimation of a material limit. The energy load limit currently forseen for the ITER divertor targets is $0.5 \mathrm{MJ} / \mathrm{m}^{2}$ for ELM pulses with $0.5 \mathrm{~ms}$ duration, based on results from numeric simulations and experimental studies $[1,8,9,10]$. Among other devices, the 
Triniti divertor material test facility QSPA-T is used for the qualification of plasma facing components during transient heat loads as expected during ELMs and disruptions in ITER [9]. The actual temporal shape of the ELM has an effect on the allowable energy load limit. Here, we characterize the ELM shape by a rise and a decay time, c.f. Fig. 2. Since the ELM rise time $\tau_{\text {rise }}$ scales with the convective parallel time $\tau_{\|}\left(\tau_{\|}=\mathrm{L}_{\mathrm{c}} / \mathrm{c}_{\mathrm{s}}\right.$, with $\mathrm{L}_{\mathrm{c}}$ : connection length, $\mathrm{c}_{\mathrm{s}}$ : ion sound speed in the SOL) $[2,11,12]$, the absolute time scales for JET and ITER are expected to be nearly identical. For JET a connection length of $\mathrm{L}_{\mathrm{c}}=64 \mathrm{~m}$ and a pedestal temperature of $\mathrm{T}_{\text {ped }}=1 \mathrm{keV}$ results in a parallel time $\tau_{\|} \sim 210 \mu \mathrm{s}$, for ITER the expected pedestal temperature of $T_{\text {ped }}=4 \mathrm{keV}$ and $\mathrm{L}_{\mathrm{c}}=120 \mathrm{~m}$ give $\tau_{\|} \sim 200 \mu \mathrm{s}$. In Fig. 2, the temporal power shape in the Triniti divertor material test facility QSPA-T $[9,10]$ is plotted with a JETELM power evolution measured by the IR camera. The rise time of $\tau_{\text {rise }}=250 \mu$ s is very similar, the decay time $\tau_{\text {decay }}$ is a little longer for the JET ELM. In Fig. 3 the rise and decay times for all ELMs in the database as measured by the IR camera for a variety of discharge conditions are plotted. The ELMs within a time window (in total 61 time windows) are represented by a data point and an uncertainty interval calculated from the standard deviation of the ELM times within the respective time window. The observed rise times are in the range between $200 \mu \mathrm{s}$ and $500 \mu \mathrm{s}$. The decay times are typically 1.5 to 5 times longer than the corresponding rise times.

The assumptions for ITER ELMs (rise time $\tau_{\text {rise,ITER }} \sim 250 \mu$ s and decay time $\tau_{\text {decay,ITER }}=250$ $500 \mu \mathrm{s})$ are therefore conservative, but the observed decay time distribution in JET ELMs is significantly larger than the ITER assumption, $\tau_{\text {decay }}>\tau_{\text {decay,ITER }}$. The ratio of the ELM deposited energy in the decay phase to that in the rise phase shows a variation from 1 to 5 , thus more energy is deposited in the decay phase (for the ITER design a ratio between 1 and 2 is assumed).

\section{ELM resolved power load scaling}

In Fig. 4 the wetted area for ELM peak and inter-ELM times are plotted. For the ELM wetted area, the data in each of the 60 time windows are grouped into energy bands of $1 \%$ relative ELM size and the mean as well as the standard deviation is calculated for each of these groups. This way, the ELM wetted area can be calculated for a larger variety of ELM sizes within one time window without losing the ELM size as a parameter. The magnitude of the ELM energy loss was found to order the ELM wetted area in a series of discharges with a 
scan in TF-ripple [13] and in IR measurements in a DIII-D discharge with a plasma current sweep [14]. This tendency can also be observed in the larger data set presented here: the minimum ELM wetted area increases with the ELM size. In the data base we find wetted areas between $0.6 \mathrm{~m}^{2}$ and $1.6 \mathrm{~m}^{2}$, corresponding to mid plane integral deposited power widths of $7 \mathrm{~mm}$ and $18 \mathrm{~mm}$, respectively.

As yet, no other clear scaling parameter could be identified. The coloured lines are fits to the data grouped by plasma current ranges. The increase of the ELM wetted area with ELM size is observed for all these plasma current groups. However, the scatter inside these groups is rather large and the fits show no obvious ordering with plasma current. We note, that the fitted slopes increase with plasma current. Similar analysis was performed for a grouping in $\mathrm{q}_{95}$ bands, but also here the ELM wetted area shows no clearer dependence.

For the inter-ELM wetted area, shown in Fig. 4 as dots, we find a slight decrease with increasing ELM size. The increase in scatter of the inter-ELM wetted area in phases with small relative ELM sizes $<2 \%$ is presumably caused by an increasing amount of ELM compound phases mistakenly considered as inter-ELM profiles by the automated analysis software. The average inter-ELM wetted area varies from $0.2 \mathrm{~m}^{2}$ to $0.5 \mathrm{~m}^{2}$. These areas translate into mid plane integral power widths of $2.5 \mathrm{~mm}$ to $6 \mathrm{~mm}$.

The power broadening varies between 1.5 (ELM wetted area equal to the inter-ELM wetted area) and 6. The target wetted area is found to show a relatively large broadening in the presence of large ELMs and a moderate broadening in conditions with smaller ELMs as shown in Fig. 4.

In Fig. 5 (a) the ELM energy load versus the ELM size is shown. The minimum energy load $\varepsilon$ is increasing by a factor of 5 for an increase in relative ELM size of a factor of 10 . The energy load displays a large scatter, which might partly be caused by the uncertainty of the ELM decay time estimation in the presence of compound ELM phases after the main ELM. The scatter in the data could also reflect (yet hidden) dependencies on plasma parameters and discharge conditions. One parameter on which the energy load appears to be sensitive is the deuterium fuelling rate: In the whole data base 32 (of 61 time windows in total) are fuelled with less than $\mathrm{G}=1 \cdot 10^{21} \mathrm{el} / \mathrm{s}$ (electrons per second), 49 are fuelled with less than $\mathrm{G}=10 \cdot 10^{21}$ $\mathrm{el} / \mathrm{s}$ and 59 are fuelled with less than $\mathrm{G}=20 \cdot 10^{21} \mathrm{el} / \mathrm{s}$. Some of the largest energy loads in the data set (denoted by coloured symbols) are found in three discharges, which form a deuterium fuelling scan with otherwise very similar plasma parameters. The fuelling rate during the steady discharge phases with type-I ELM activity was increased from $\mathrm{G}=2 \cdot 10^{21} \mathrm{el} / \mathrm{s}$ to $\mathrm{G}=25 \cdot 10^{21} \mathrm{el} / \mathrm{s}$ and $\mathrm{G}=35 \cdot 10^{12} \mathrm{el} / \mathrm{s}$ on a shot-by-shot basis. The fuelling rates of the latter 
two time windows are the largest in the data base. The wetted areas and the peak heat fluxes are shown in Fig. 5 (b,c).

The maximum target-integrated power during an ELM (ELM peak power) increases roughly linearly with the ELM size (not shown), as does the ELM wetted area (cf. Fig. 5 (c)), but the average ELM peak heat flux stays constant (Fig. 5(b)). Some ELMs at the intermediate deuterium fuelling rate (\#74372) show an even higher peak heat flux for a reduced ELM size with respect to the reference discharge (\#74366). Thus, for these three discharges, the increase in the wetted area is caused by the increase in deposited ELM power.

The average temporal ELM decay times are increasing with the fuelling rate: $800+/-100 \mu \mathrm{s}$ (\#74366), $1200+/-140 \mu$ s (\#74372) and 1650 +/-300 $\mu$ s (\#74373). The longer decay times can partly explain the increased energy load, cf. Fig. 5(a), since the energy load is integrated over a longer time period. Hence, the energy load increase is caused by the increase in the wetted area (due to the spatial average around the peak heat flux in the definition of the energy load in Eq. (1)) and by the slower temporal decay. The inter-ELM wetted area is decreasing from $\mathrm{A}=0.35 \mathrm{~m}^{2}$ at $\sim 2 \%$ ELM size to $\mathrm{A}=0.2 \mathrm{~m}^{2}$ at $7 \%$ ELM size.

\section{Discussion}

The relative ELM size estimated from the drop of the stored diamagnetic energy $\left(\mathrm{W}_{\mathrm{MHD}}\right)$ apparently orders the data estimated from the IR measurements, like the increase of the wetted area with the ELM size, rather well. An ELM resolved power balance $\Delta \mathrm{W}_{\text {target }}=\Delta \mathrm{W}_{\mathrm{MHD}}+\Delta \mathrm{W}_{\mathrm{in}}-\Delta \mathrm{W}_{\text {rad }}$, taking into account the radiated power loss estimated by bolometry $\Delta \mathrm{W}_{\text {rad }}$, the drop in stored energy $\Delta \mathrm{W}_{\mathrm{MHD}}$ and the total energy input from heating $\Delta \mathrm{W}_{\text {in }}$ on a $4 \mathrm{~ms}$ time scale (constrained by the time resolution of the bolometer system) on average shows a good agreement. Apart from the different magnitude in ELM size, the general findings (i.e., ELM broadening) are similar for an energy loss estimate $500 \mu$ s after the drop instead of $4 \mathrm{~ms}$.

One result from the power balance analysis is that the scatter of $\Delta \mathrm{W}_{\mathrm{MHD}}$ for ELMs during a steady discharge phase is approximately twice as large as the scatter of energy measured with the IR system $\Delta \mathrm{W}_{\text {target }}$. The scatter in the data might partly be associated with the applied semi-automated ELM detection, since the algorithm also accounts for ELMs in compound 
phases. The advantage of this approach is a more objective study of ELM power loads compared to data bases with (typically few) hand-selected ELMs.

The results on ELM time scales prove the assumptions on ELM time scales in ITER to be conservative estimates, since the observed rise times are as expected also for ITER. It remains to be confirmed in material test facility experiments, that a different temporal evolution of the ELM power load with more energy in the decay phase is compatible with the adopted energy load limit of $0.5 \mathrm{MJ} / \mathrm{m}^{2}$ in $500 \mu$ s for transients.

The observed inter-ELM integral deposited power widths as narrow as $2.5 \mathrm{~mm}$ (Fig. 4) are compatible with previously reported values measured by other diagnostics [12]. Results reported from Langmuir probe measurements in JET indicated an ELM broadening [15] although it could not be confirmed by the old IR camera system, presumably due to the limited spatial and temporal resolution of that system. With the new IR system, the broadening can be confirmed to exist and is found to be in the range between 1.5 and 6 , depending on plasma conditions.

The ELM energy loads expected for ITER are estimated using the assumption of an ELM wetted area equal to the inter-ELM wetted area as an upper limit (broadening of 1) [1]. The observation in JET and in DIII-D [14], that the power broadening scales with the ELM size is an indication, that such behaviour might also be expected for ITER (although more data from other fusion devices and a multi-machine scaling are necessary to increase confidence in such extrapolation). If this assumption is valid, larger ELMs might be tolerable in ITER due to the increased wetted area. In Ref. [1], the consequences for ITER ELMs assuming a broadening of 4 instead of 1 are estimated and it is found that natural ELMs might be acceptable for the divertor targets for higher plasma currents Ip: the operation regime without active ELM control would be extended from a maximum $\mathrm{I}_{\mathrm{p}}=6 \mathrm{MA}$ to a maximum of $\mathrm{I}_{\mathrm{p}}=9.5 \mathrm{MA}$. For higher plasma currents a scenario with fully controlled ELMs is still required, even with the assumption of an beneficial broadening.

It is not clear, whether it will be possible to establish a stable scenario with large ELMs in conjunction with a large ELM wetted area in ITER. Even if this was the case, the less protected first wall materials outside the divertor target might also get a larger share of the energy due to the enhanced radial transport. Moreover, the inter-ELM wetted area shows a tendency to narrow in regimes with larger ELMs (Fig. 4), which would increase the interELM power load on the divertor targets. For the case of a scenario with small type-I ELMs it could be feasible to spread the power load by sweeping the strike lines and thereby effectively wetting a larger divertor target area. 
A caveat might be the finding that ELMs reduced in size by means of deuterium fuelling, may have a similar or even larger energy load compared to the natural ELMs. To a certain extent this effect can be explained by the longer decay phases in the temporal ELM shapes leading to a longer integration time for calculating the energy load. It remains to be investigated by material test facilities, whether a temporal ELM shape with a much longer decay phase might be less problematic in terms of surface erosion. We note, that for a constant integration time and same start values, the estimated energy load generally will be larger for a slower temporal decay than for a faster decaying curve. The observation that the average ELM peak heat flux is constant in the deuterium fuelling scan, cf. Fig. 5(b), thus indicates that the energy load is higher also for an estimate with the same (constant) integration time used for all ELMs instead of $\tau_{\text {decay. }}$

In almost all analyzed deuterium fuelling scans we observe constant average ELM peak heat fluxes. One exception, where the ELM peak heat flux is decreasing on average with increasing fuelling rate, is reported in Ref. [16]. In these experiments, the maximum deuterium fuelling rate $(\mathrm{G}=151021 \mathrm{el} / \mathrm{s})$ is lower than the intermediate rate $(\mathrm{G}=251021 \mathrm{el} / \mathrm{s})$ in the fueling scan reported here. One might speculate, whether a fuelling rate threshold for this effect exists. A common feature in fuelled discharges is the increase in ELM decay time with the increasing fuelling level.

With the generally observed increase of the ELM frequency in discharges with enhanced deuterium fuelling, the increased number of small ELMs (in terms of diamagnetic loss) might result in a larger target erosion due to their smaller wetted area compared to a smaller number of larger natural ELMs with a larger deposition area. The evaluation of ELM mitigation scenarios should therefore include an assessment of the wetted area and energy loads $[16,17]$.

\section{Conclusions}

Following the installation of a fast infrared camera at the JET tokamak with a high spatial and temporal resolution, ELM-resolved power loads have been studied A data base including power and energy loads obtained from the analysis of the IR measurements during more than 6000 individual ELMs (and their pre-ELM periods) has been set up. The IR data is validated by means of discharge integrated divertor thermo-couple measurements and to Langmuir probe data.

The ELM size estimated from the diamagnetic energy is not uniquely determining the ELM energy load at the divertor target. ELM power broadening is observed to widen with 
increasing ELM size for a variety of discharge conditions. The inter-ELM wetted area is found to become smaller for scenarios with larger type-I ELMs. Furthermore, the ELM shape was characterized. The ELM rise times are found to be in the expected range, but the deposited energy during the ELM decay is larger than assumed for the ITER design. It remains to be confirmed in material test facility experiments, that a different temporal evolution of the ELM power load with more energy in the decay phase is compatible with the adopted energy load limit of $0.5 \mathrm{MJ} / \mathrm{m}^{2}$ in $500 \mu \mathrm{s}$ for transients. Further input from other machines is required for a multi-machine scaling necessary for a solid extrapolation to ITER.

\section{Acknowledgements}

This work was supported by EURATOM and carried out within the framework of the European Fusion Development Agreement. The views and opinions expressed herein do not necessarily reflect those of the European Commission.

\section{References}

[1] A Loarte et al. in Fusion Energy 2010 (Proc. 23rd Int. Conf. Daejeon, 2010) (Vienna: IAEA) CD-ROM file ITR/1-4 and http://www-naweb.iaea.org/napc/physics/FEC/FEC2010/html/index.htm

[2] A. Loarte et al., Plasma Phys. Control. Fusion 45 (2003) 1549.

[3] S. Jachmich et al. , 36th EPS Conf. on Controlled Fusion and Plasma Physics (Sofia, Bulgaria) (Europhysics Conference Abstracts Vol 33E) (2009) P-2.159.

[4] T. Eich et al., J. Nucl. Mater. (2011) in press (doi:10.1016/j.jnucmat.2010.11.079).

[5] A.Herrmann et al., J. Nucl.Materials, 337-339 (2005) 907.

[6] A. Herrmann, Phys. Scr. T128 (2007) 234.

[7] A. Loarte et al., J. Nucl.Materials 266-269 (1999) 587.

[8] J. Linke et al., J. Nucl. Mater. 367-370 (2007) 1422.

[9] N. Klimov et al., J. Nucl. Materials 390-391 (2009) 721,

[10] N. Klimov et al., J. Nucl. Mater. (2011) in press (doi:10.1016/j.jnucmat.2011.01.013).

[11] T. Eich et al., Plasma Phys. Contr. Fusion 47 (2005) 815.

[12] W. Fundamenski et al., Nucl. Fusion 45 (2005) 950.

[13] Thomsen H. et al, 36th EPS Conf. on Controlled Fusion and Plasma Physics (Sofia, Bulgaria) (Europhysics Conference Abstracts Vol 33E) (2009) P-2.164.

[14] M. Jakubowski et al., Nucl. Fusion 49 (2009) 095013.

[15] S. Jachmich et al., J. Nucl. Mater. 363-365 (2007) 1050.

[16] S. Jachmich et al., J. Nucl. Mater. (2011) in press. 
[17] E. de la Luna et al. in Fusion Energy 2010 (Proc. 23rd Int. Conf. Daejeon, 2010) (Vienna: IAEA) CD-ROM file EXC/8-4 and http://www-naweb.iaea.org/napc/physics/FEC/FEC2010/html/index.htm.

[18] R. Sartori et al., Plasma Phys. Control. Fusion 46 (2004) 723.

Figures:

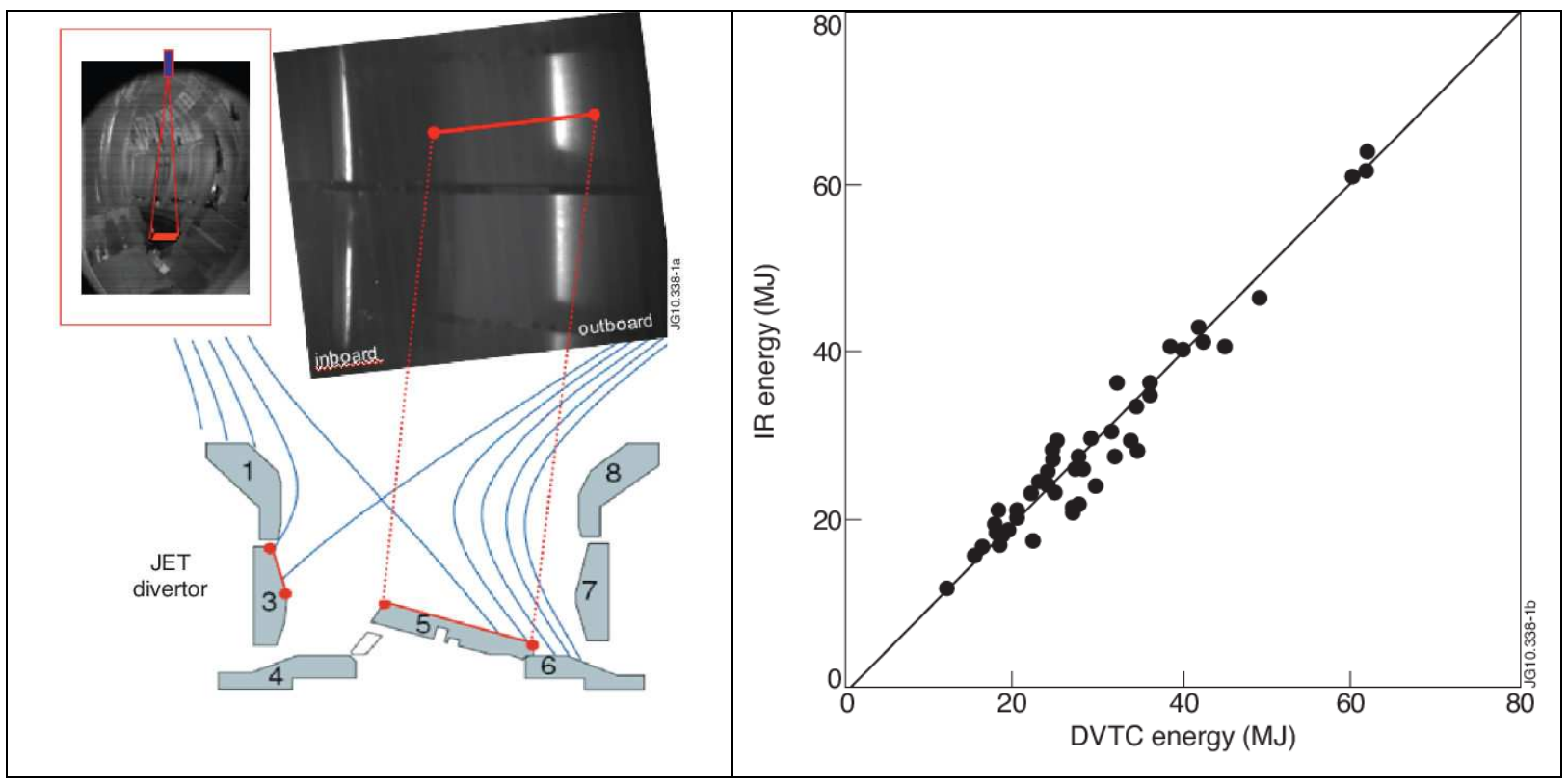

FIG 1: (a) Setup of the newly installed IR camera in the JET tokamak. The red line across the outbouard divertor indicates the location of the $4 \times 150$ pixels strip used for the analysis. (b) Comparison of IR and thermocouple (DVTC) estimated total energies for 52 discharges in the data base. 


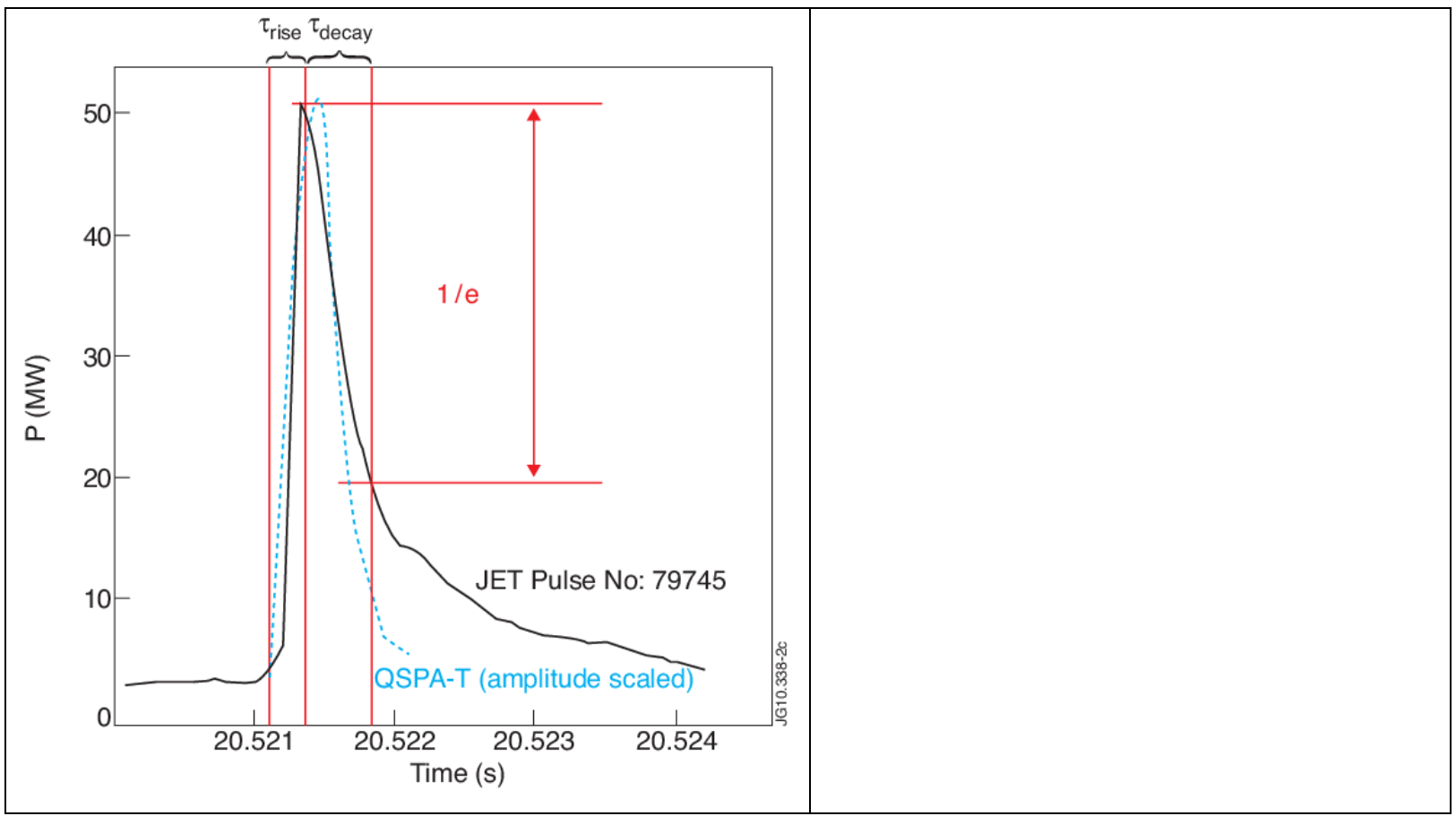

FIG 2: Time scales of a JET ELM and the power shape of the Triniti plasma gun QSPA-T for testing plasma facing materials for the ITER divertor scaled in amplitude to match the peak power of the JET-ELM. 


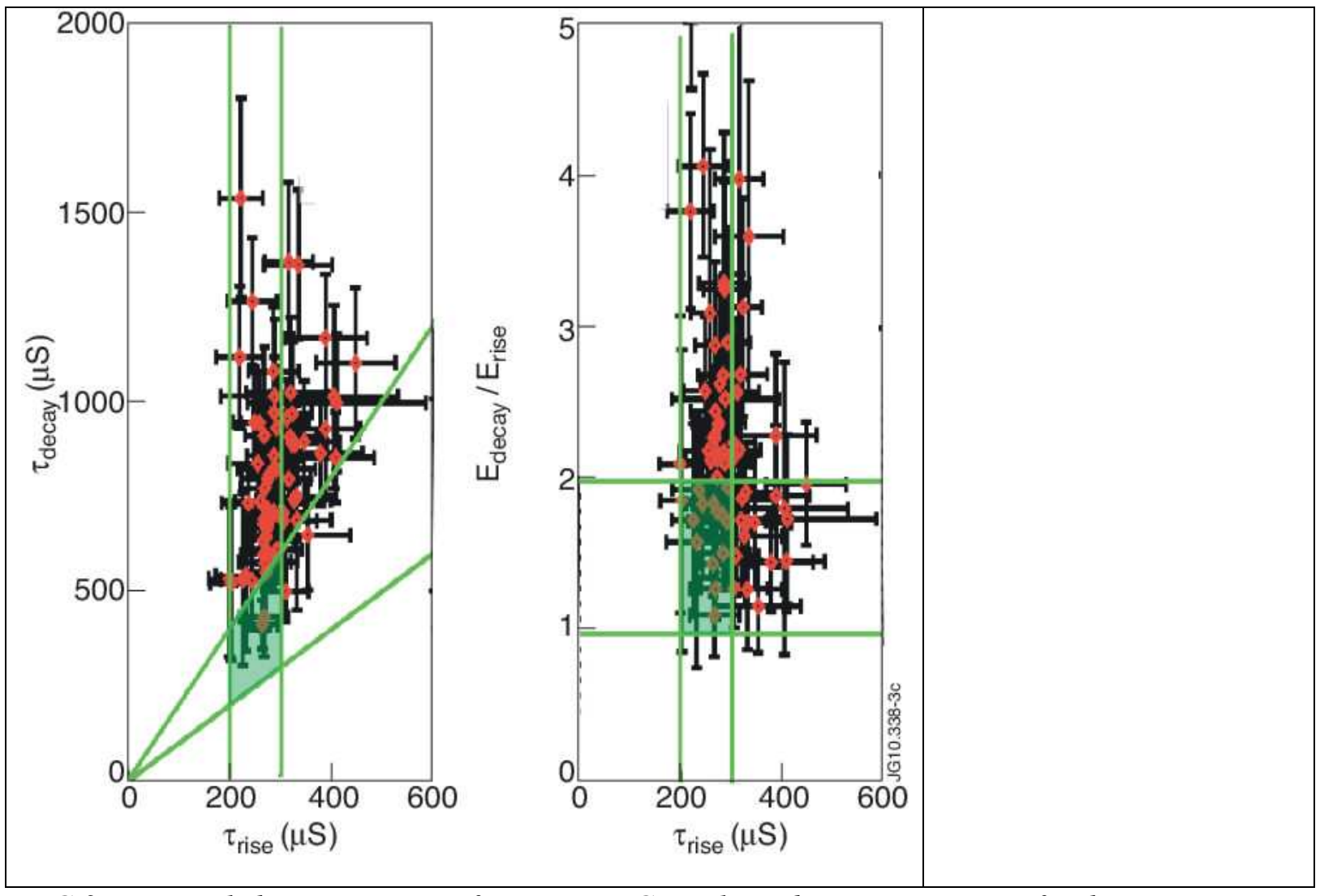

FIG.3. Temporal characterization of JET ELMs. Green lines denote assumptions for the ITER design.Left:ELM decay time versus ELM rise time. Right: Ratio of the ELM energy deposited during decay and rise phase versus ELM rise time. 


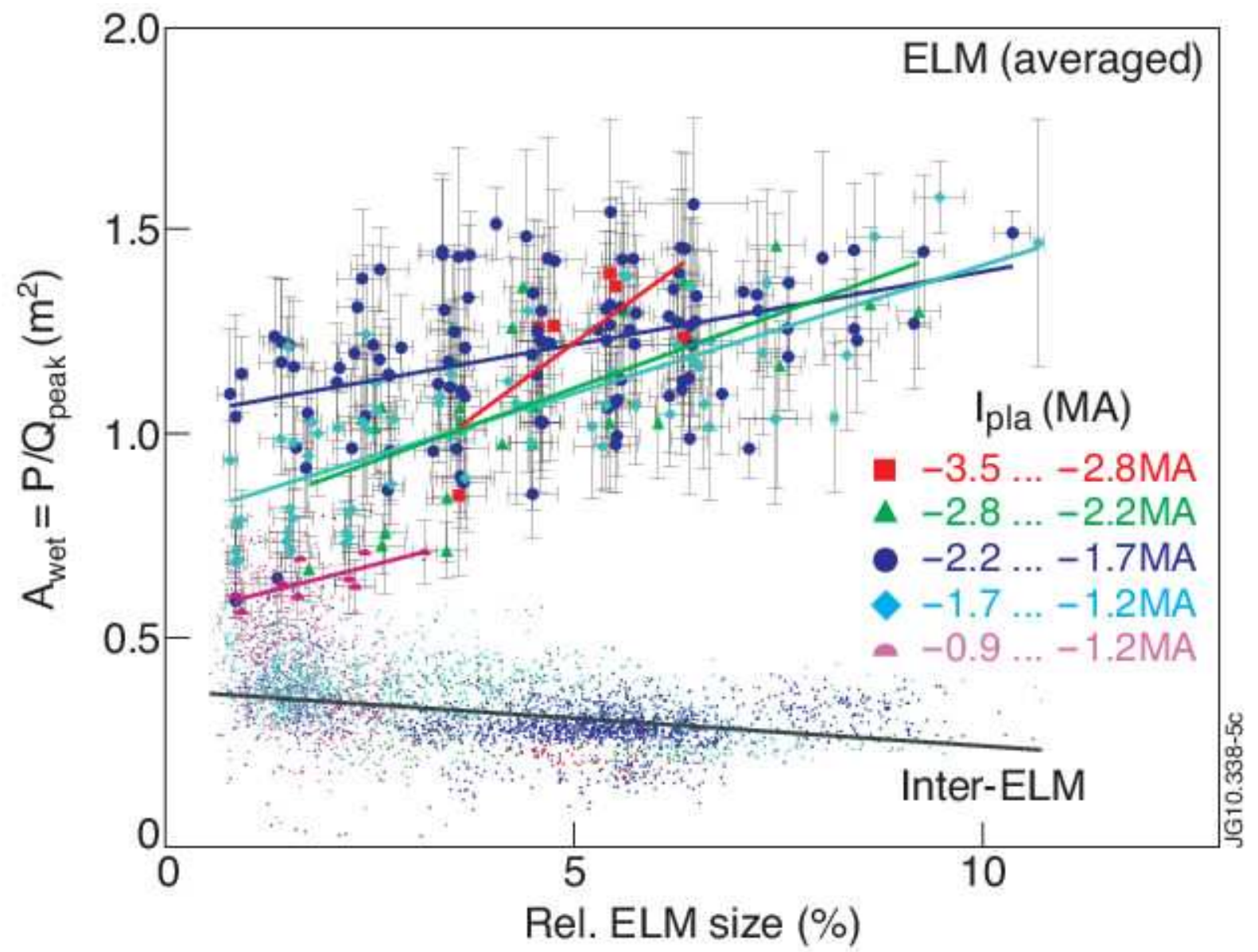

FIG 4: Database of wetted area versus the ELM size normalized to $W_{M H D}$. Different colours indicate plasma current intervals (negative plasma currents $I_{p}$ correspond to the regular magnetic configuration in JET) and solid lines denote linear fits to the wetted area in the respective interval. 


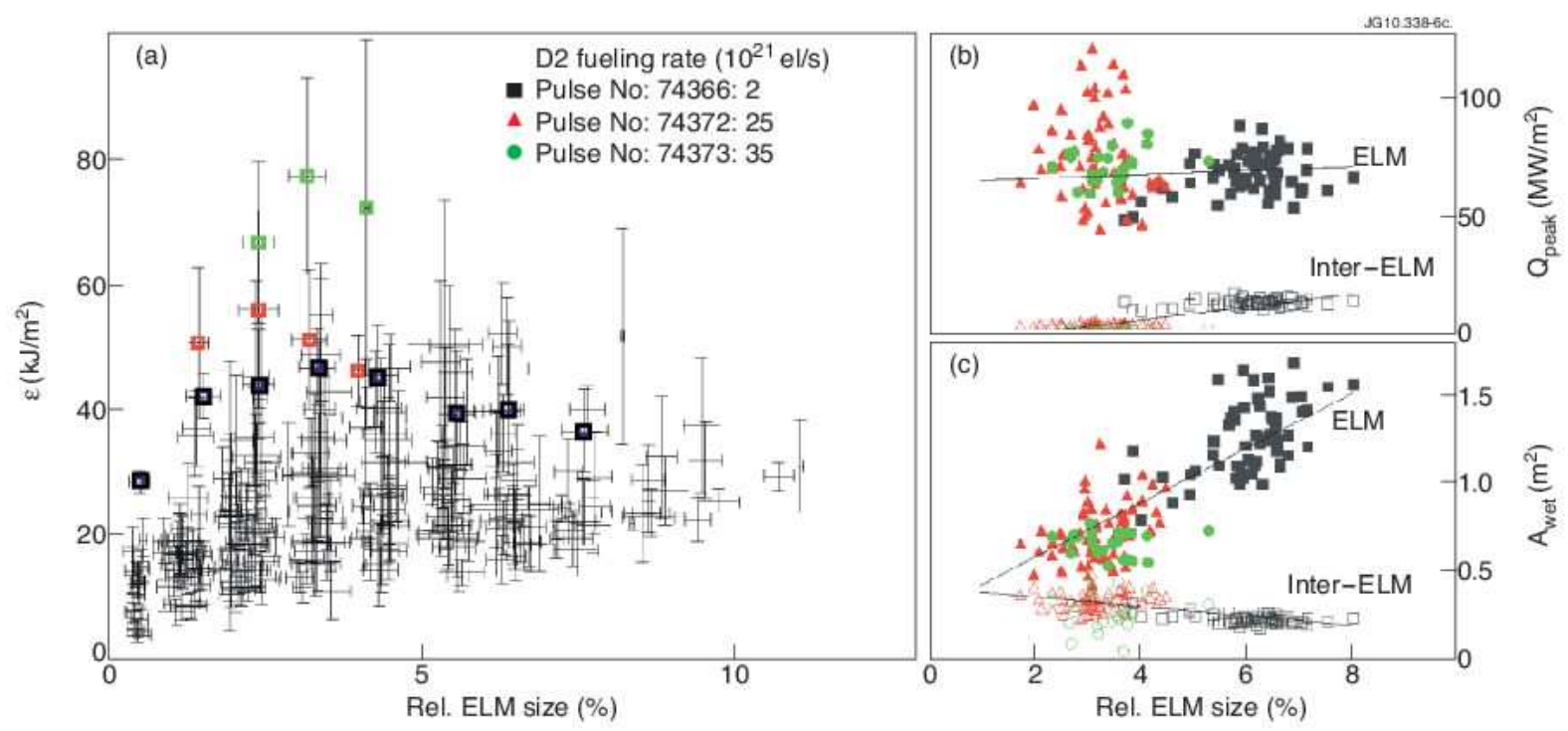

FIG 5: (a) Energy load versus ELM size normalized to $W_{M H D}$ for the data base. Colours denote values from a deuterium fuelling scan. (b) Corresponding peak heat fluxes and (c) wetted areas. Solid lines are linear fits to the data. 
